\title{
Packet-Reliability-Based Decode-and-Forward Distributed Space-Time Shift Keying
}

\author{
S. Sugiura, S. Chen and L. Hanzo \\ School of ECS, University of Southampton, SO17 1BJ, UK, Tel: +44-23-8059-3125, Fax: +44-23-8059-4508 \\ Email: \{ss07r,sqc,1h\}@ecs.soton.ac.uk, http://www-mobile.ecs.soton.ac.uk
}

\begin{abstract}
Motivated by the recent concept of Space-Time Shift Keying (STSK), we propose a novel cooperative STSK scheme, which is capable of achieving a flexible rate-diversity tradeoff, in the context of cooperative space-time transmissions. More specifically, in our cooperative STSK scheme each Relay Node (RN) activates Decode-and-Forward (DF) transmissions, depending on the success or failure of Cyclic Redundancy Checking (CRC). We propose a novel bit-to STSK mapping rule, where according to the input bits, one of the $Q$ pre-assigned dispersion vectors is activated to implicitly convey $\log _{2} Q$ bits, which are transmitted in combination with the classic $\log _{2} \mathcal{L}$-bit modulated symbol. Additionally, we introduce a beneficial dispersion vector design, which enables us to dispense with symbol-level Inter-Relay Synchronization (IRS). Furthermore, the Destination Node (DN) is capable of jointly detecting the signals received from the source-destination and relay-destination links, using a low-complexity single-stream-based Maximum Likelihood (ML) detector, which is an explicit benefit of our Inter-Element Interference (IEI)-free system model. More importantly, as a benefit of its design flexibility, our cooperative STSK arrangement enables us to adapt the number of the RNs, the transmission rate as well as the achievable diversity order.
\end{abstract}

\section{INTRODUCTION}

In recent years, cooperative Space-Time Codes (STCs) [1], [2] have been extensively investigated due to their potential to achieve a high transmit diversity gain, where a collection of distributed nodes act as a Virtual Antenna Array (VAA), which are positioned sufficiently far apart for experiencing uncorrelated Multiple-Input Multiple-Output (MIMO) channels. Furthermore, this VAA arrangement enables us to avoid the employment of multiple RF chains at each node. Most of the previously-proposed cooperative STCs assumed perfect Inter-Relay Synchronization (IRS), although it is a challenging task to acquire symbol-level timing synchronization between the distributed Relay Nodes (RNs). However, as noted in [3], the asynchronous transmissions of the RNs may destroy the STC's orthogonality, leading to a severe performance degradation. For the sake of effectively combating this IRS problem, a number of asynchronous cooperative STCs were proposed in [4], [5].

Recently, the sophisticated concept of Spatial Modulation (SM) [6], [7] was proposed for co-located MIMO elements, where only one of the $M$ available transmit antennas is activated within each symbol interval, which serves as an additional implicit means of conveying information, over and above the conventional symbol constellation. As a benefit, in contrast to V-BLAST, the resultant system model does not suffer from any Inter-Element Interference (IEI). Therefore, also in contrast to V-BLAST, low-complexity single-antenna-based Maximum Likelihood (ML) detection can be utilized at the receiver instead of the joint detection of multiple streams. More specifically, it was found in [6], [7] that SM has the potential of outperforming other MIMO arrangements, such as V-BLAST and Alamouti's STBC schemes. Considering that the SM scheme can be operated without perfect Inter-Antenna Synchronization (IAS), our proposition is that this arrangement may also be suitable for cooperative communication scenarios, although no cooperative SM scheme has been presented

The financial support of the EU under the auspices of the Optimix project and of the EPSRC UK is gratefully acknowledged. The work of S. Sugiura was also sponsored in part by the Toyota Central Research \& Development Laboratories, Inc., Japan. in the open literature. However, since SM was not designed for providing transmit diversity gain, increasing the number of RNs would not increase the cooperative diversity order.

More recently, in [8], [9] the novel concept of Space-Time Shift Keying (STSK) was invented, where space-time codewords are generated by activating one out of $Q$ space-time dispersion matrices, rather than one out of $M$ antenna elements as in the SM scheme [6], [7]. The STSK scheme is capable of achieving a flexible tradeoff between the attainable diversity and throughput, hence outperforming other MIMO arrangements, such as OSTBCs, BLAST and SM schemes. ${ }^{1}$ Furthermore, since no Inter-Channel Interference (ICI) is imposed by the STSK receiver, low-complexity single-stream-based ML detection may be invoked.

Motivated by the recent concept of STSK [8], the novel contribution of this paper is that we propose a cooperative STSK scheme, which acts as a unified shift keying architecture designed for achieving a useful cooperative space-time diversity. Our scheme is capable of activating an arbitrary number of RNs, as well as of appropriately adjusting both the transmission rate and the diversity gain. More specifically, each $R N$ is a constituent part of our distributed Decode-and-Forward (DF) scheme, where one out of $Q$ pre-assigned dispersion vectors is activated and transmitted within each block duration for transmitting a PSK/QAM symbol. Moreover, each relay participates in the cooperative regime in a distributed manner, namely without negotiating with the SN or RNs, hence achieves a substantial reduction of the overhead compared to coordinated DF schemes. Furthermore, by exploiting the fact that no IEI is imposed at the $D N$ in our cooperative STSK scheme, we derive a single-streambased low-complexity ML detector, whose complexity is comparable to that of Orthogonal Space-Time Block Coding (OSTBC) schemes [11], [12]. We also demonstrate that by appropriately designing the $Q$ pre-assigned dispersion vectors, the RNs of the cooperative STSK scheme is capable of dispensing with the requirement of perfectly synchronizing their transmissions with other RNs, which leads to a high robustness against IRS errors limited to a fraction of the symbol duration.

\section{System Model}

This section describes our cooperative STSK scheme. As seen in Fig. 1, we consider a two-phase relay network, which is constituted by a single Source Node ( $\mathrm{SN}), M$ RNs and a DN, each having a single antenna element. We note that the proposed scheme can be readily extended to the multiple-antenna-element assisted DN scenario. Here, it is assumed that a Time Division Multiple Access (TDMA) protocol is used and that the cooperating relays attempt to loosely-synchronize their timings under the BS's control, ${ }^{2}$ but nevertheless, we will demonstrate that the proposed regime is resilient against IRS errors. We also assume that each node is operated in a half-duplex mode,

\footnotetext{
${ }^{1}$ To expound a little further, in [10] it was demonstrated that the generalized STSK scheme subsumes many other MIMO arrangements, such as OSTBCs, BLAST, LDCs and SM schemes.

${ }^{2}$ The synchronization accuracy achieved by the Medium Access Control (MAC) layer is typically lower than the symbol-synchronized simultaneous transmissions of the relays.
} 


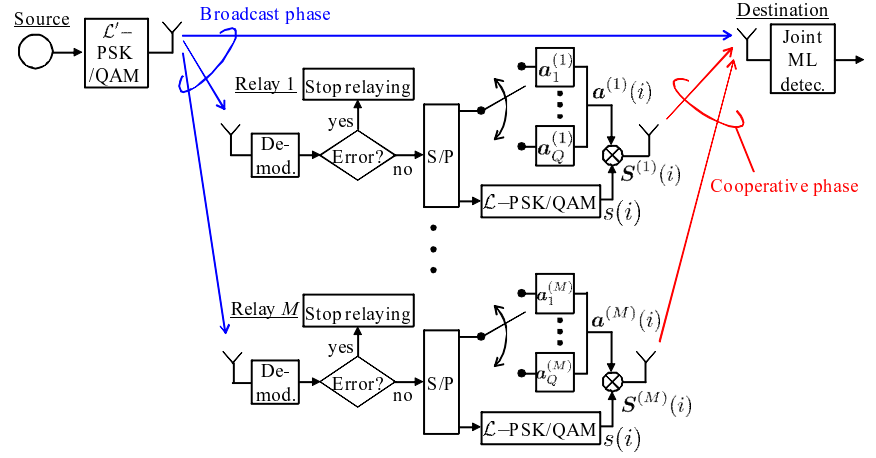

Fig. 1. Schematic of our distributed STSK scheme assisted by selective DF relaying.

either receiving or transmitting in a given time slot. Additionally, for the sake of enabling the CRC at the RNs, frame-based rather than symbol-based transmissions are carried out, although this leads to an increased detection delay.

\section{A. Source Model}

During the broadcast phase of Fig. 1, the SN transmits its information to the $M$ RNs as well as to the DN. As noted in [2], the SN first attaches Cyclic Redundancy Check (CRC) bits to its information bits in order for the RNs to be able to detect the potential decoding errors and hence to avoid the propagation of $\mathrm{DF}$ errors to the DN. Then the CRC-encoded bits are mapped to the $\mathcal{L}^{\prime}$-constellation point PSK/QAM scheme in order to generate the symbols $\boldsymbol{S}_{\mathrm{s}}(i)=\left[s_{1}(i), \cdots, s_{b}(i)\right]^{T} \in \mathcal{C}^{b \times 1}$, where $i$ represents the block index and $b \log _{2} \mathcal{L}^{\prime}$ bits are transmitted in each block. Let us assume that the $\mathrm{SN}$ transmits the symbols $\boldsymbol{S}_{\mathrm{s}}(i)$ over $b$ time slots, and the corresponding signals received at the $m$ th $\mathrm{RN}$ as well as at the $\mathrm{DN}$ are given by

$$
\boldsymbol{Y}_{\mathrm{sr}}^{(m)}(i)=h_{\mathrm{sr}}^{(m)}(i) \boldsymbol{S}_{\mathrm{s}}(i)+\boldsymbol{N}_{\mathrm{r}}^{(m)}(i)
$$

and

$$
\boldsymbol{Y}_{\mathrm{sd}}(i)=h_{\mathrm{sd}}(i) \boldsymbol{S}_{\mathrm{s}}(i)+\boldsymbol{N}_{\mathrm{d}}(i),
$$

respectively, where the channel coefficients $h_{\mathrm{sr}}^{(m)}$ and $h_{\mathrm{sd}}(i)$ obey the complex-valued Gaussian distributions of $\mathcal{C N}\left(0, \sigma_{\mathrm{sd}}^{2}\right)$ and of $\mathcal{C N}\left(0, \sigma_{\text {sr }}^{2}\right)$, while each component of the noise vectors $\boldsymbol{N}_{\mathrm{r}}^{(m)}(i)$ and $\boldsymbol{N}_{\mathrm{d}}(i)$ is a complex-valued Gaussian variable of $\mathcal{C N}\left(0, N_{0}\right)$. Furthermore, $N_{0}$ represents the noise variance and $L_{\mathrm{f}}$ denotes the number of $b \log _{2} \mathcal{L}^{\prime}$-bit signal blocks, which are successively transmitted in each single transmission frame during the broadcast phase.

\section{B. Relay Model}

Let us now describe the cooperative phase of Fig. 1, where the $M$ RNs employ CRC-activated DF transmission with the aid of our cooperative STSK scheme. Let us consider the $m$ th RN to be the node of interest, which first decodes the received signals $\boldsymbol{Y}_{\mathrm{sr}}^{(m)}(i)$ of Eq. (1). If any decoding error is identified by the CRC, the RN refrains from relaying the signals to the $\mathrm{DN}$ without requiring any negotiation with the other nodes. By contrast, if there are no decoding errors, the $m$ th relay re-encodes the decoded bits and transmits them using the cooperative STSK scheme as follows. The $b \log _{2} \mathcal{L}^{\prime}$ decoded bits per $b$-slot block are Serial-to-Parallel (S/P) converted to $\log _{2} Q$ and $\log _{2} \mathcal{L}$ bits, assuming the relationship of $b \log _{2} \mathcal{L}^{\prime}=\log _{2}(Q \cdot \mathcal{L})$. Here, we will represent each of the corresponding S/P converted bits as $(q, l)$ in decimal representation. Then, as shown in Fig. 1, the bits
TABLE I

EXAMPLE of $m$ TH Relay's STSK Modulation SCHEME, MapPing 3 Bits PER SPACE-TIME Block, With THE AID OF BPSK CONSTELLATION

\begin{tabular}{|rll||c|r|r|}
\hline \multicolumn{4}{|c||}{} & \multicolumn{3}{c|}{$Q=4, \mathcal{L}=2$} \\
\hline \multicolumn{3}{|c||}{ Input bits } & \multicolumn{3}{c|}{ Mapped symbols } \\
\hline \multicolumn{2}{|c||}{$(q, l)$} & $\boldsymbol{a}^{(m)}(i)$ & $s(i)$ & \multicolumn{1}{c|}{$\boldsymbol{S}_{\mathrm{r}}^{(m)}(i)$} \\
\hline 00 & $\boldsymbol{a}_{1}^{(m)}$ & +1 & $\boldsymbol{a}_{1}^{(m)}$ \\
00 & 1 & 1 & $\boldsymbol{a}_{1}^{(m)}$ & -1 & $-\boldsymbol{a}_{1}^{(m)}$ \\
01 & 1 & 0 & $\boldsymbol{a}_{2}^{(m)}$ & +1 & $\boldsymbol{a}_{2}^{(m)}$ \\
01 & 1 & 1 & $\boldsymbol{a}_{2}^{(m)}$ & -1 & $-\boldsymbol{a}_{2}^{(m)}$ \\
10 & 1 & 0 & $\boldsymbol{a}_{3}^{(m)}$ & +1 & $\boldsymbol{a}_{3}^{(m)}$ \\
10 & 1 & 1 & $\boldsymbol{a}_{3}^{(m)}$ & -1 & $-\boldsymbol{a}_{3}^{(m)}$ \\
11 & 0 & $\boldsymbol{a}_{4}^{(m)}$ & +1 & $\boldsymbol{a}_{4}^{(m)}$ \\
$\underbrace{11}_{\log _{2} Q}$ & $\underbrace{}_{\log _{2} \mathcal{L}}$ & $\boldsymbol{a}_{4}^{(m)}$ & -1 & $-\boldsymbol{a}_{4}^{(m)}$ \\
\hline
\end{tabular}

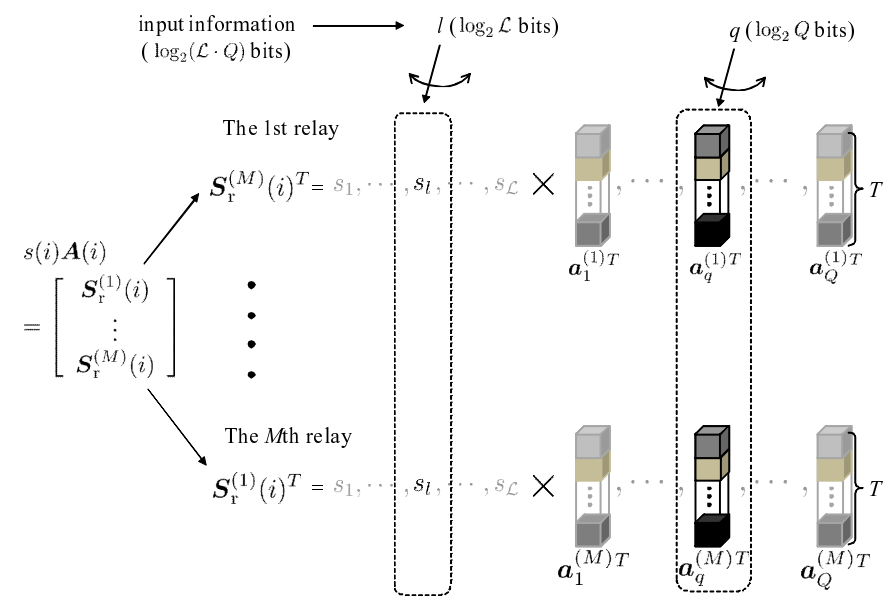

Fig. 2. Structure of the cooperative space-time codeword $s(i) \boldsymbol{A}(i)$ in Eq (5).

decoded at the $m$ th $\mathrm{RN}$ are mapped to a $T$-length symbol vector $\boldsymbol{S}_{\mathrm{r}}^{(m)}(i) \in \mathcal{C}^{1 \times T}$, which is given by

$$
\boldsymbol{S}_{\mathrm{r}}^{(m)}(i)=s(i) \boldsymbol{a}^{(m)}(i),
$$

where according to the input bits $q, \boldsymbol{a}^{(m)}(i)$ is selected from the $Q$ pre-assigned dispersion vectors $\boldsymbol{a}_{q^{\prime}}^{(m)} \in \mathcal{C}^{1 \times T}\left(q^{\prime}=1,2, \cdots, Q\right)$, while $s(i)$ is selected from an $\mathcal{L}$-point PSK/QAM constellation according to the input bits $l$. Similarly to the broadcast phase, $L_{\mathrm{f}}$ denotes the number of space-time blocks successively transmitted in each single transmission frame during the cooperative phase. We note that the input bit-dependent selection of a dispersion vector from a set of $Q$ provides an additional implicit means of transmitting $\log _{2} Q$ bits of information, similarly to the antenna selection philosophy of SM [6], [7]. To elaborate a little further, the $m$ th relay's bit-to-symbol mapping regime of our cooperative STSK scheme employing $Q=4$ and $\mathcal{L}=2$ is shown in Table I. Given $B=\log _{2}(Q \cdot \mathcal{L})$ input bits per block, there are several potential combinations of $Q$ and $\mathcal{L}$, for instance, $(Q, \mathcal{L})=(8,1),(4,2),(2,4)$ and $(1,8)$ for the abovementioned case of conveying $\log _{2}(Q \cdot \mathcal{L})=3$ input bits.

Having generated the STSK-modulated signal vector $\boldsymbol{S}_{\mathrm{r}}^{(m)}(i)$ as illustrated in Fig. 2, the corresponding signals received at the DN may be expressed as

$$
\begin{aligned}
\boldsymbol{Y}_{\mathrm{rd}}(i) & =\sum_{m=1}^{M} \alpha_{m} h_{\mathrm{rd}}^{(m)}(i) \boldsymbol{S}_{\mathrm{r}}^{(m)}(i)+\boldsymbol{N}_{\mathrm{d}}^{\prime}(i) \\
& =s(i) \boldsymbol{H}_{\mathrm{rd}}(i) \boldsymbol{A}(i)+\boldsymbol{N}_{\mathrm{d}}^{\prime}(i),
\end{aligned}
$$


where we have

$$
\begin{aligned}
\boldsymbol{H}_{\mathrm{rd}}(i) & =\left[\alpha_{1} h_{\mathrm{rd}}^{(1)}(i), \cdots, \alpha_{M} h_{\mathrm{rd}}^{(M)}(i)\right] \in \mathcal{C}^{1 \times M}, \\
\boldsymbol{A}(i) & =\left[\begin{array}{c}
\boldsymbol{a}^{(1)}(i) \\
\vdots \\
\boldsymbol{a}^{(M)}(i)
\end{array}\right] \in \mathcal{C}^{M \times T} .
\end{aligned}
$$

Here, $\alpha_{m} \in\{1,0\}$ represents the activation/deactivation of the $m$ th relay, where $\alpha_{m}$ is 0 , if any decoding error is identified by the CRC scheme of the $m$ th relay. Otherwise $\alpha_{m}$ is set to 1 . Furthermore, the RN-DN channel coefficients $h_{\mathrm{rd}}^{(m)}(i)(m=1, \cdots, M)$ and the destination's noise components $\boldsymbol{N}_{\mathrm{d}}^{\prime}(i)$ follow the complex-valued Gaussian distributions of $\mathcal{C N}\left(0, \sigma_{\text {rd }}^{2}\right)$ and $\mathcal{C N}\left(0, N_{0}\right)$, respectively. In order to maintain a unity transmission power per time slot, the $M \cdot Q$ pre-assigned dispersion vectors $\boldsymbol{a}_{q}^{(m)}(1 \leq m \leq M, 1 \leq q \leq$ $Q$ ) have to satisfy

$$
\operatorname{tr}\left(\boldsymbol{A}_{q^{\prime}} \boldsymbol{A}_{q^{\prime}}^{H}\right)=T\left(q^{\prime}=1, \cdots, Q\right),
$$

where $\operatorname{tr}()$ represents the trace of a matrix and

$$
\boldsymbol{A}_{q^{\prime}}=\left[\begin{array}{c}
\boldsymbol{a}_{q^{\prime}}^{(1)} \\
\vdots \\
\boldsymbol{a}_{q^{\prime}}^{(M)}
\end{array}\right] \in \mathcal{C}^{M \times T} .
$$

It is implied in Eq. (5) that the RNs typically have to synchronize with each other within a fraction of the symbol duration, owing to the requirement of their simultaneous transmissions. However, as mentioned above, it is a challenging task to acquire accurate IRS. Therefore, in order to relax this IRS-related limitation, hereby we impose a further constraint on the $Q$ dispersion matrices of $\boldsymbol{A}_{q^{\prime}}\left(q^{\prime}=1, \cdots, Q\right)$ in addition to the power constraint of Eq. (8). More specifically, $\boldsymbol{A}_{q^{\prime}}$ is generated by ensuring that in each column of $\boldsymbol{A}_{q^{\prime}}$ only one component has a complex non-zero value, while the others become zero. By obeying this constraint, only one of the $M$ RNs transmits its signal during each symbol interval, and hence we can avoid the requirement of symbol-synchronized simultaneous relay transmissions. The design criterion of the $Q$ dispersion matrices employed in this contribution will be detailed in Section IV.

Additionally, the normalized throughput per each of the $b$ time slots or per cooperative STSK symbol duration for the broadcast phase is $R_{1}$ and that of the cooperative phase is $R_{2}$, which are given by

$$
\begin{array}{ll}
R_{1}=\log _{2} \mathcal{L}^{\prime} & {[\mathrm{bits} / \mathrm{symbol}]} \\
R_{2}=\frac{\log _{2}(Q \cdot \mathcal{L})}{T} & {[\mathrm{bits} / \mathrm{symbol}],}
\end{array}
$$

leading to the total normalized throughput of

$$
R=\frac{b R_{1}+T R_{2}}{2(b+T)}=\frac{\log _{2}(Q \cdot \mathcal{L})}{b+T}[\mathrm{bits} / \mathrm{symbol}] .
$$

It can be seen from Eq. (11) that upon increasing either the number of dispersion vectors $Q$ or the classic PSK/QAM constellation size $\mathcal{L}$, the transmission rate of the cooperative phase increases.

Moreover, the maximum achievable transmit diversity order of the cooperative STSK is upper-bounded by $\min (M, T)$, according to the well-known pairwise-error probability analysis based on the Chernoff upper bound [13], whose detailed derivation is omitted in this paper owing to space limitations.

To elaborate a little further, as explicitly mentioned in [7], the SM scheme developed for co-located MIMO arrangements enables the transmitter to dispense with symbol-level IAS, since in the SM scheme only a single $\mathrm{AE}$ is activated during each symbol duration. Similarly, due to the above-mentioned constraint of imposing a sparse structure on the dispersion matrices of our cooperative
Asynchronous STSK (ASTSK) scheme, only a single RN is activated within each symbol duration, hence exhibiting robustness against IRS errors provided that they are limited to a fraction of the symbolduration. We note that the potential IRS error may severely degrade the performance of conventional cooperative STCs, which require symbol-level IRS, as investigated in [3]. On the other hand, when the IRS error exceeds the symbol duration, a severe impairment may be imposed also on our cooperative ASTSK scheme, since the structure of the STSK codeword is destroyed. In order to combat this limitation associated with high IRS errors, we may be able to incorporate the Loosely-Synchronized (LS)-code aided Space-Time Spreading (STS) technique [5] into our cooperative STSK scheme, although the detailed investigations will be left for our future studies owing to space-limitations.

\section{IEI-FREE JOINT ML DETECTION AT THE DESTINATION RECEIVER}

At the DN, the directly transmitted signals of Eq. (2) and the relayed signals of Eq. (5) are jointly detected using a low-complexity single-stream ML detector.

Firstly, by applying the vectorial stacking operation $v e c()$ to both sides of Eq. (5), we arrive at the linearized relay-destination system's output in the form of [13]

$$
\overline{\boldsymbol{Y}}_{\mathrm{rd}}(i)=\overline{\boldsymbol{H}}_{\mathrm{rd}}(i) \chi \boldsymbol{K}(i)+\overline{\boldsymbol{N}}_{\mathrm{d}}(i),
$$

where we have

$$
\begin{array}{rlrl}
\overline{\boldsymbol{Y}}_{\mathrm{rd}}(i) & =\operatorname{vec}\left[\boldsymbol{Y}_{\mathrm{rd}}(i)\right] & & \in \mathcal{C}^{T \times 1}, \\
\overline{\boldsymbol{H}}_{\mathrm{rd}}(i) & =\boldsymbol{I} \otimes \boldsymbol{H}_{\mathrm{rd}}(i) & & \in \mathcal{C}^{T \times M T}, \\
\overline{\boldsymbol{N}}^{\prime}{ }_{\mathrm{d}}(i) & =\operatorname{vec}\left[\boldsymbol{N}_{\mathrm{d}}^{\prime}(i)\right] & \in \mathcal{C}^{T \times 1}, \\
\boldsymbol{\chi} & =\left[\operatorname{vec}\left(\boldsymbol{A}_{1}\right), \cdots, \operatorname{vec}\left(\boldsymbol{A}_{Q}\right)\right] & \in \mathcal{C}^{M T \times Q},
\end{array}
$$

and

$$
\boldsymbol{K}(i)=[\underbrace{0, \cdots, 0}_{q-1}, s(i), \underbrace{0, \cdots, 0}_{Q-q}]^{T} \in \mathcal{C}^{Q \times 1} .
$$

Furthermore, $\boldsymbol{I}$ is the identity matrix and $\otimes$ is the Kronecker product. It is worth mentioning that the linearized relay-destination system model of Eq. (13) does not contain any IEI, because the equivalent signal vector $\boldsymbol{K}(i)$ has only a single non-zero symbol component, similarly to SM [6], [7].

Finally, the joint system model, combining the broadcast phase of Eq. (2) and the cooperative phase of Eq. (13), may be formulated as

$$
\begin{aligned}
\hat{\boldsymbol{Y}}(i) & =\left[\begin{array}{c}
\boldsymbol{Y}_{\mathrm{sd}}(i) \\
\overline{\boldsymbol{Y}}_{\mathrm{rd}}(i)
\end{array}\right] \in \mathcal{C}^{(b+T) \times 1} \\
& =\hat{\boldsymbol{H}}(i) \hat{\boldsymbol{S}}(i)+\hat{\boldsymbol{N}}(i),
\end{aligned}
$$

where we have

$$
\begin{aligned}
\hat{\boldsymbol{H}}(i) & =\left[\begin{array}{c}
h_{\mathrm{sd}}(i) \boldsymbol{I}_{\underline{b}} \\
-\mathbf{0}^{-}+\overline{\boldsymbol{H}}_{\mathrm{rd}}^{-}(i) \bar{\chi}
\end{array}\right] \in \mathcal{C}^{(b+T) \times(b+Q)} \\
\hat{\boldsymbol{S}}(i) & =\left[\begin{array}{c}
\boldsymbol{S}_{\mathrm{s}}(i) \\
\boldsymbol{K}(i)
\end{array}\right], \hat{\boldsymbol{N}}(i)=\left[\begin{array}{c}
\boldsymbol{N}_{\mathrm{d}}(i) \\
\overline{\boldsymbol{N}}^{\prime}(i)
\end{array}\right]
\end{aligned}
$$

Let us then consider the conditional probability of

$$
\begin{aligned}
P\left(\hat{\boldsymbol{Y}}(i) \mid \hat{\boldsymbol{H}}(i), \hat{\boldsymbol{S}}^{(q, l)}\right) & =\frac{1}{\left(\pi N_{0}\right)^{b+T}} \\
& \times \exp \left(-\frac{\left\|\hat{\boldsymbol{Y}}(i)-\hat{\boldsymbol{H}}(i) \hat{\boldsymbol{S}}^{(q, l)}\right\|^{2}}{N_{0}}\right),
\end{aligned}
$$


where

$$
\hat{\boldsymbol{S}}^{(q, l)}=\left[\begin{array}{c}
\boldsymbol{S}_{\mathrm{s}}^{(q, l)} \\
\boldsymbol{K}^{(q, l)}
\end{array}\right] \in \mathcal{C}^{(b+Q) \times 1}
$$

with

$$
\boldsymbol{K}^{(q, l)}=[\underbrace{0, \cdots, 0}_{q-1}, s_{l}, \underbrace{0, \cdots, 0}_{Q-q}]^{T} .
$$

Here, $s_{l}$ denotes the $l$ th constellation point of $\mathcal{L}$-PSK/QAM, employed during the cooperative phase and $\boldsymbol{S}_{\mathrm{s}}^{(q, l)}$ represents the modulated symbols of the broadcast phase, corresponding to the bits of the set $(q, l)$.

Then, the optimal ML detector of our cooperative STSK scheme may be formulated with the assistance of [7] as

$$
\begin{aligned}
(\hat{q}, \hat{l}) & =\arg \max _{q, l} P\left(\hat{\boldsymbol{Y}}(i) \mid \hat{\boldsymbol{H}}(i), \hat{\boldsymbol{S}}^{(q, l)}\right) \\
& =\arg \min _{q, l}\left\|\hat{\boldsymbol{Y}}(i)-\hat{\boldsymbol{H}}(i) \hat{\boldsymbol{S}}^{(q, l)}\right\|^{2} \\
& =\arg \min _{q, l}\left(\left\|\boldsymbol{Y}_{\mathrm{sd}}(i)-h_{\mathrm{sd}}(i) \boldsymbol{S}_{\mathrm{s}}^{(q, l)}\right\|^{2}\right. \\
& \left.+\left\|\overline{\boldsymbol{Y}}_{\mathrm{rd}}(i)-s_{l}\left(\overline{\boldsymbol{H}}_{\mathrm{rd}}(i) \chi\right)_{q}\right\|^{2}\right),
\end{aligned}
$$

where $\left(\overline{\boldsymbol{H}}_{\mathrm{rd}}(i) \boldsymbol{\chi}\right)_{q}$ is the $q$ th column of $\overline{\boldsymbol{H}}_{\mathrm{rd}}(i) \boldsymbol{\chi}$. The first term of Eq. (27) indicates the detection of the source-destination signals, while the second term corresponds to that of the relay-destination signals, where all the signal components are independent of each other and hence no IEI is imposed.

The computational complexity per bit imposed by calculating Eq. (27) per bit may be evaluated in terms of the number of real-valued multiplications, which is given by

$$
\frac{4 T^{2}+6 T Q \mathcal{L}+4 \mathcal{L}^{\prime}+2 b Q \mathcal{L}}{\log _{2}(Q \cdot \mathcal{L})} .
$$

This complexity is as low as those of the OSTBC [12] and SM schemes [7] used in an identical cooperative scenario. In the rest of this paper, we employ the parameter-based system notation of $\operatorname{STSK}(M, T, Q)$ for the cooperative phase.

\section{Performance Results}

In this section we provide our performance results, comparing different DF scenarios as well as different cooperative schemes. In line with [5], we considered independent Rayleigh block-fading environments, having the geometrical distance-reduction based channel gains of $\sigma_{\mathrm{sd}}^{2}=1, \sigma_{\mathrm{sr}}^{2}=4$ and $\sigma_{\mathrm{rd}}^{2}=2$, which remains constant over $(b+T)$ STSK symbol durations. Furthermore, the $\mathrm{SN}$ was assumed to employ QPSK modulation, which indicates $\mathcal{L}^{\prime}=4$.

According to the previous studies of Linear Dispersion Codes (LDCs) [14], there exists several potential approaches to the optimization of the dispersion matrix set $\boldsymbol{A}_{q^{\prime}}\left(q^{\prime}=1, \cdots, Q\right)$, such as the capacity maximization criterion [15] and pairwise error probability minimization [16]. In this contribution, we employ the well-known rank- and determinant-criterion of [15] in order to attain the maximum achievable diversity order as well as a high coding gain. The dispersion-vector sets, which were obtained by random search and were employed in our simulations, are shown in the Appendix. The basic system parameters employed for our simulations are listed in Table II.

Fig. 3 shows the achievable BER performance of our cooperative $\operatorname{STSK}(2,2,4)$ scheme, employing QPSK modulation both at the SN and at the RNs, where the normalized transmission rate was $R=1.0$ bits/symbol. Here, we compared three different DF schemes, namely the perfect decision based DF, the conventional DF and the proposed
TABLE II

BASIC SYSTEM PARAMETERS OF THE COOPERATIVE STSK $(M, T, Q)$ SCHEME

\begin{tabular}{lr}
\hline \hline Number of RNs & $M=2-4$ \\
Number of AEs at each node & $N=1$ \\
Symbol durations per block & $T=2,3$ \\
Number of dispersion matrices & $Q=2,4,8,16$ \\
Modulation during broadcast phase & $\mathcal{L}^{\prime}-$ PSK/QAM \\
Modulation during cooperative phase & $\mathcal{L}-$ PSK/QAM \\
Relaying scheme & CRC-activated selective DF \\
Channels & Frequency-flat Rayleigh fading \\
Channel's coherence-time & $\tau=1$ block duration \\
Geometrical channel gains & $\left(\sigma_{\text {sd }}^{2}, \sigma_{\text {sr }}^{2}, \sigma_{\text {rd }}^{2}\right)=(1,4,2)$ \\
Detector & Joint ML detector of Eq. $(27)$ \\
\hline \hline
\end{tabular}

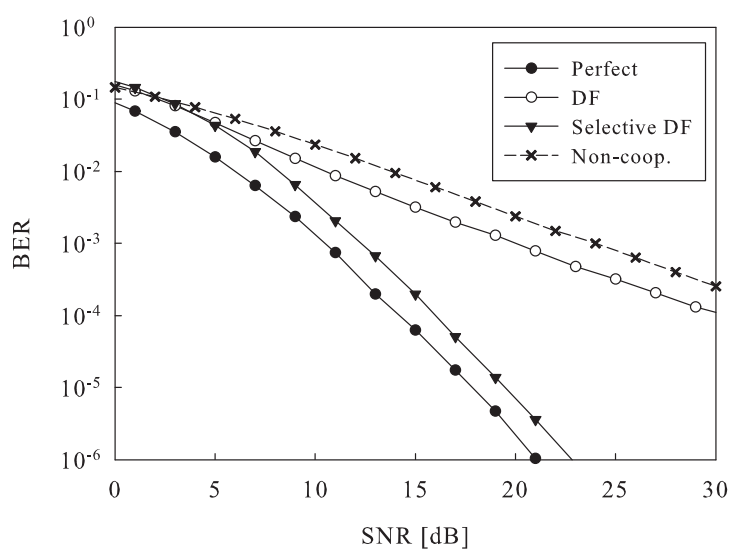

Fig. 3. Achievable BER performance of our QPSK-modulated cooperative $\operatorname{STSK}(2,2,4)$ system, comparing different DF relaying schemes, such as the perfect DF scheme having no information loss, the conventional DF scheme and the CRC-activated DF scheme. We also characterized the non-cooperative scenario employing BPSK modulation.

CRC-activated DF schemes, where the perfect DF scheme assumed having perfect source-relay channels, hence imposing no errors by the relays' decoders, while in the conventional DF scheme all the $M$ RNs were assumed to join the cooperative transmission regime, regardless of the presence or absence of decoding errors. We also plotted the BER curve of the corresponding non-cooperative scenario, assuming the employment of BPSK modulation at the SN. Observe in Fig. 3 that the perfect and the selective DF schemes attained a transmit diversity order of three, hence both outperformed the conventional DF scheme and the non-cooperative scenario. Here, it should be emphasized that the selective DF scheme achieved a reduced-overhead distributed operation at each $\mathrm{RN}$. On the other hand, the BER curves of the conventional DF scheme and of the noncooperative scenario did not exhibit any additional transmit diversity gain.

In order to provide further insights, in Figs. 4 and 5 we compared our cooperative STSK and ASTSK schemes to the cooperative OSTBC arrangements [12], having the corresponding bandwidth efficiency. Here, we CRC-activated selective DF relaying for all the simulated scenarios. Fig. 4 investigated the scenario of a normalized transmission rate of $R=1.0 \mathrm{bits} / \mathrm{symbol}$, where $M=4 \mathrm{RNs}$ were considered. Observe in Fig. 4 that all the three cooperative schemes achieved a useful diversity gains in comparison to the noncooperative scenario. The cooperative STSK scheme outperformed the cooperative OSTBC scheme, as predicted from the results characterized by the co-located STSK arrangements [8]. Additionally, the 


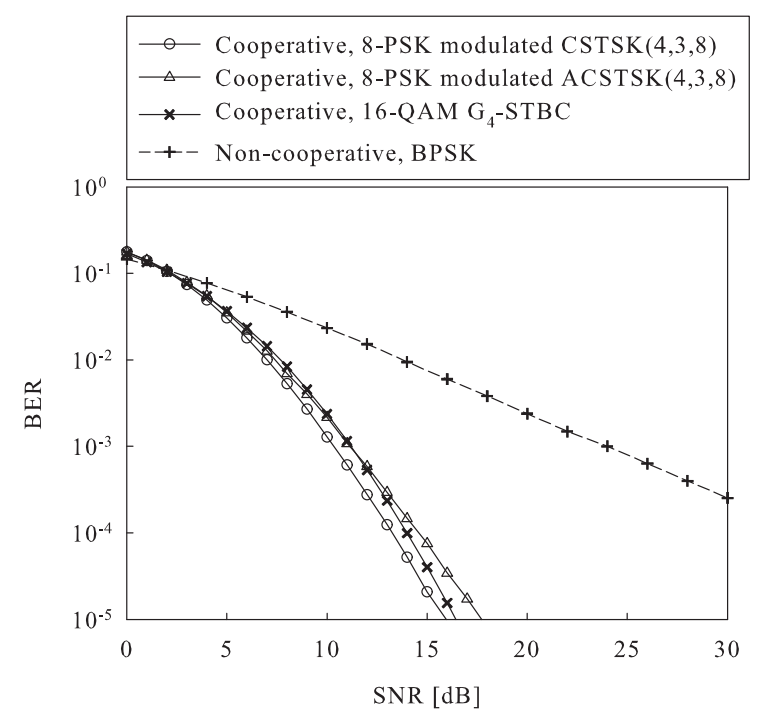

Fig. 4. Achievable BER performance of our $\mathcal{L}=8$ PSK-modulated cooperative $\operatorname{STSK}(4,3,8)$ and $\mathcal{L}=8$ PSK-modulated cooperative $\operatorname{ASTSK}(4,3,8)$ schemes obeying the architecture of Fig. 1, while $\mathcal{L}^{\prime}=8$ PSK was employed for the SN. The corresponding BER curves of the cooperative $\mathrm{G}_{4}-\mathrm{STBC}$ scheme as well as of the BPSK-modulated non-cooperative scheme were also calculated as benchmarkers.

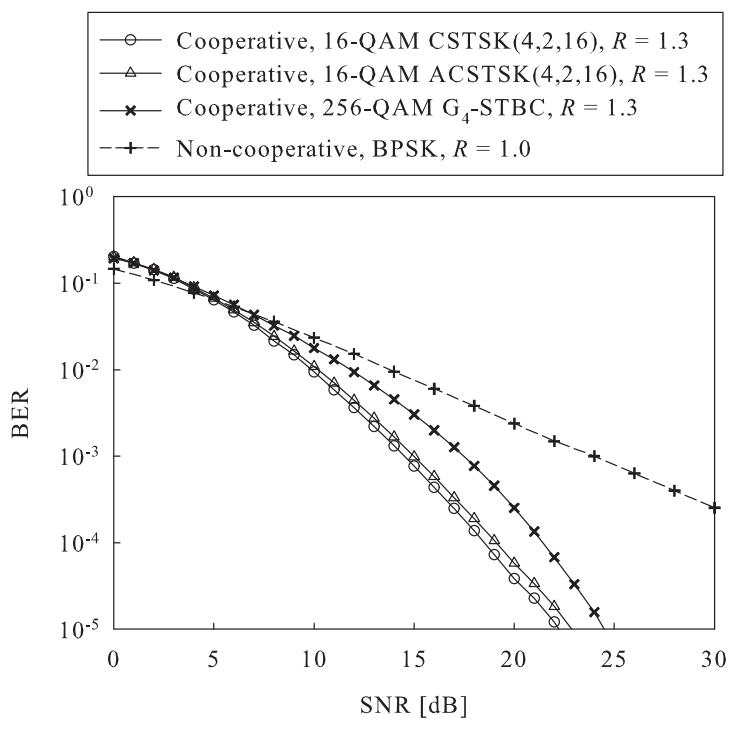

Fig. 5. Achievable BER performance of our $\mathcal{L}=16$ QAM cooperative $\operatorname{STSK}(4,2,16)$ and $\mathcal{L}=16 \mathrm{QAM}$ cooperative $\operatorname{ASTSK}(4,2,16)$ schemes obeying the architecture of Fig. 1, while $\mathcal{L}^{\prime}=4$ PSK was employed for the SN. The corresponding BER curves of the $\mathcal{L}=256$ QAM cooperative $\mathrm{G}_{4}$-STBC scheme as well as BPSK-modulated non-cooperative scheme were also calculated as benchmarkers.

cooperative ASTSK scheme exhibited a slightly lower performance than those of the cooperative STSK and OSTBC schemes, due to the restricted dispersion matrix structure. Nevertheless, the cooperative ASTSK scheme's benefit of dispensing with symbol-level IRS may be especially useful for the scenario suffering from a rapid topology change, where perfect IRS is hard to achieve.

Furthermore, when increasing the normalized transmission rate $R$, the performance advantage of our STSK and ASTSK schemes becomes more explicit, as observed in Fig. 5, which correspond to the scenarios of the normalized transmission rate of $R=1.3$ bits/symbol. To expound a little further, it was found in Fig. 5 that our STSK and ASTSK schemes outperformed the corresponding BER of the cooperative OSTBC scheme. This is mainly owing to the fact that the cooperative OSTBC scheme is typically required to employ power-hungry high order modulation, in order to attain an increased transmission rate.

\section{Conclusions}

Motivated by the recent STSK concept, we proposed a novel cooperative STSK scheme, where each RN uses CRC-activated DF relaying employing STSK during the cooperative phase of Fig. 1, which is capable of attaining an attractive cooperative diversity gain. Here, the RNs do not require symbol-synchronization owing to the additional restriction which we imposed on the dispersion vector design. At the receiver, the received signals of the direct sourcedestination link and the relay-destination links are jointly detected using IEI-free low-complexity single-stream ML detection. More importantly, owing to its design flexibility, our cooperative STSK arrangement enables us to adapt the number of RNs, the transmission rate as well as the achievable diversity order, depending on the associated system requirements and channel conditions.

\section{REFERENCES}

[1] J. Laneman and G. Wornell, "Distributed space-time-coded protocols for exploiting cooperative diversity in wireless networks," IEEE Transactions on Information Theory, vol. 49, no. 10, pp. 2415-2425, 2003.

[2] T. Wang, A. Cano, G. Giannakis, and J. Laneman, "High-performance cooperative demodulation with decode-and-forward relays," IEEE Transactions on Communications, vol. 55, no. 7, pp. 1427-1438, 2007.

[3] R. C. Palat, A. Annamalai, and J. H. Reed, "Accurate bit-error-rate analysis of bandlimited cooperative OSTBC networks under timing synchronization errors," IEEE Transactions on Vehicular Technology, vol. 58, no. 5, pp. 2191-2200, 2009.

[4] M. Sharp, A. Scaglione, and B. Sirkeci-Mergen, "Randomized cooperation in asynchronous dispersive links," IEEE Transactions on Communications, vol. 57, no. 1, pp. 64-68, 2009.

[5] S. Sugiura, S. Chen, and L. Hanzo, "Cooperative differential spacetime spreading for the asynchronous relay aided CDMA uplink using interference rejection spreading code," IEEE Signal Processing Letters, vol. 17 , no. 2 , pp. $117-120,2010$.

[6] R. Mesleh, H. Haas, S. Sinanovic, C. Ahn, and S. Yun, "Spatial modulation," IEEE Transactions on Vehicular Technology, vol. 57, no. 4 pp. 2228-2242, 2008.

[7] J. Jeganathan, A. Ghrayeb, and L. Szczecinski, "Spatial modulation: optimal detection and performance analysis," IEEE Communications Letters, vol. 12, no. 8, pp. 545-547, 2008.

[8] S. Sugiura, S. Chen, and L. Hanzo, "Coherent and differential spacetime shift keying: a dispersion matrix approach," IEEE Transactions on Communications, accepted for publication.

[9] —_, "Space-time shift keying: A unified MIMO architecture," in IEEE Global Telecommunications Conference, Miami, Florida, USA, 6-10 December 2010, pp. 1-5.

[10] — - "A unified MIMO architecture subsuming space shift keying, OSTBC, BLAST and LDC," in IEEE 72nd Vehicular Technology Conference (VTC2010-Fall), Ottawa, Canada, 6-9 September 2010, pp. 1-5.

[11] S. Alamouti, "A simple transmit diversity technique for wireless communications," IEEE Journal on Selected Areas in Communications, vol. 16 no. 8, pp. 1451-1458, 1998.

[12] V. Tarokh, H. Jafarkhani, and A. Calderbank, "Space-time block coding for wireless communications: performance results," IEEE Journal on Selected Areas in Communications, vol. 17, no. 3, pp. 451-460, 1999.

[13] R. W. Heath Jr and A. J. Paulraj, "Linear dispersion codes for MIMO systems based on frame theory," IEEE Transactions on Signal Processing, vol. 50, no. 10, pp. 2429-2441, 2002.

[14] B. Hassibi and B. Hochwald, "High-rate codes that are linear in space and time," IEEE Transactions on Information Theory, vol. 48, no. 7, pp. 1804-1824, 2002.

[15] L. Hanzo, O. Alamri, M. El-Hajjar, and N. Wu, Near-Capacity MultiFunctional MIMO Systems: Sphere-Packing, Iterative Detection and Cooperation. John Wiley and IEEE Press, 2009, 714 pages.

[16] J. Wang, X. Wang, and M. Madihian, "On the optimum design of space-time linear-dispersion codes," IEEE Transactions on Wireless Communications, vol. 4, no. 6, pp. 2928-2938, 2005. 